\title{
Detecção precoce do câncer do colo do útero no Brasil: um estudo dos indicadores de cobertura e adesão às diretrizes técnicas nacionais
}

\author{
Early detection of cervical cancer in Brazill: a study of coverage indicators and adherence to \\ national technical guidelines
}

Detección temprana del cáncer cervicouterino en Brasil: un estudio de indicadores de cobertura y adherencia a las directrices técnicas nacionales

\begin{abstract}
Resumo
Objetivo: Analisar os indicadores relacionados a cobertura e adesão às diretrizes para controle do Câncer de Colo de útero no Brasil no período de 2015 a 2020 . Método: Estudo ecológico, de abordagem quantitativa, elaborado com dados contidos no Sistema de Informação do Câncer e no Sistema de Informação Ambulatorial. Utilizou-se também dados provenientes do censo demográfico do Instituto Brasileiro de Geografia e Estatística e do Instituto Nacional de Câncer. A análise dos dados foi realizada por meio do cálculo dos indicadores no programa Excel e os resultados foram dispostos na forma de tabelas. Resultados: A Razão de exames citopatológicos do colo do útero em mulheres de 25 a 64 anos e a população feminina da mesma faixa etária no período estudado, apresentou tendência de queda em todas as regiões do país. Na proporção de exames citopatológicos do colo do útero na faixa etária alvo, verificou-se que as maiores proporções foram alcançadas entre regiões norte e sudeste, e as menores proporções nas regiões Sul e Centro Oeste. Quanto a proporção de exames citopatológicos do colo do útero sem citologia anterior, observou-se um padrão de queda em todas as regiões do Brasil e na proporção de exames citopatológicos do colo do útero com periodicidade trienal, as regiões Norte e Nordeste apresentaram os melhores desempenhos. Conclusão: As tendências de queda evidenciadas nos
\end{abstract}


indicadores de cobertura e adesão às diretrizes nacionais podem refletir no aumento da incidência, morbidade e mortalidade do câncer do colo do útero.

Palavras-chave: Neoplasias do colo do útero; Sistemas de informação em saúde; Indicadores de saúde.

\begin{abstract}
Objective: To analyze indicators related to coverage and adherence to guidelines for the control of Cervical Cancer in Brazil from 2015 to 2020. Method: Ecological study, with a quantitative approach, prepared with data contained in the Cancer Information System and in the Outpatient Information System. Data from the demographic census of the Brazilian Institute of Geography and Statistics and the National Institute of Cancer were also used. Data analysis was performed by calculating the indicators in the Excel program and the results were arranged in the form of tables. Results: The ratio of cervical cytopathological exams in women aged 25 to 64 years and the female population of the same age group in the period studied showed a downward trend in all regions of the country. Regarding the proportion of cervical cytopathological exams in the target age group, it was found that the highest proportions were reached between the North and Southeast regions, and the lowest proportions in the South and Midwest regions. As for the proportion of cervical cytopathological exams without previous cytology, a pattern of decline was observed in all regions of Brazil and in the proportion of cervical cytopathological exams with a triennial periodicity, the North and Northeast regions presented the best performances. . Conclusion: The downward trends evidenced in the indicators of coverage and adherence to national guidelines may reflect an increase in the incidence, morbidity and mortality of cervical cancer.

Keywords: Uterine cervical neoplasms; Health information systems; Health indicators.
\end{abstract}

\title{
Resumen
}

Objetivo: Analizar indicadores relacionados con la cobertura y la adherencia a las directrices para el control del Cáncer de Cuello Uterino en Brasil de 2015 a 2020. Método: Estudio ecológico, con enfoque cuantitativo, elaborado con datos contenidos en el Sistema de Información del Cáncer y en el Sistema de Información del Ambulatorio. También se utilizaron datos del censo demográfico del Instituto Brasileño de Geografía y Estadística y del Instituto Nacional del Cáncer. El análisis de datos se realizó mediante el cálculo de los indicadores en el programa Excel y los resultados se ordenaron en forma de tablas. Resultados: La relación de exámenes citopatológicos de cuello uterino en mujeres de 25 a 64 años y la población femenina del mismo grupo de edad en el período estudiado mostró una tendencia a la baja en todas las regiones del país. En cuanto a la proporción de exámenes citopatológicos cervicales en el grupo de edad objetivo, se verificó que las proporciones más altas se alcanzaron entre las regiones Norte y Sudeste, y las proporciones más bajas en las regiones Sur y Centro Oeste. En cuanto a la proporción de exámenes citopatológicos cervicales sin citología previa, se observó un patrón de descenso en todas las regiones de Brasil y en la proporción de exámenes citopatológicos cervicales con periodicidad trienal, las regiones Norte y Nordeste presentaron los mejores desempeños. Conclusión: Las tendencias a la baja evidenciadas en los indicadores de cobertura y adherencia a las guías nacionales pueden reflejar un aumento en la incidencia, morbilidad y mortalidad del cáncer de cuello uterino.

Palabras clave: Neoplasias del cuello uterino; Sistemas de Información en salud; Indicadores de salud.

\section{Introdução}

O Câncer do Colo do Útero é uma neoplasia maligna caracterizada pela replicação desordenada do epitélio de revestimento do colo do útero e o acometimento do tecido subjacente a ele, o estroma. A infecção persistente pelos tipos oncogênicos do papilomavírus humano (HPV), é fator determinante para o surgimento deste câncer, os tipos 16 e o 18 são responsáveis por cerca de 70\% dos casos. (Organização Pan-Americana da Saúde [OPAS], 2019; Instituto Nacional de Câncer [INCA], 2021a)

A história natural da doença possui desenvolvimento lento, portanto o rastreamento é uma importante ferramenta para a detecção precoce pois possibilita o reconhecimento de lesões precursoras que poderão ser tratadas e curadas antes que possam progredir para o câncer (Teixeira et al., 2021). O exame citopatológico também conhecido como Papanicolaou, é o método de rastreio mais utilizado no Brasil, deve ser realizado em mulheres na faixa etária dos 25 aos 64 anos que já tenham iniciado sua vida sexual, por dois anos seguidos, caso ambos os resultados sejam negativos, passa a ser realizado com intervalo trienal. (INCA, 2016)

Evidências apontam que quando realizado em mulheres com menos de 25 anos, o exame apresenta menor eficiência, portanto não é recomendado e não causa impacto na redução da incidência e mortalidade por câncer do colo do útero (INCA, 2016). Mesmo diante dessas fortes evidências, a falta de conhecimento da população somado ao padrão de rastreio oportunístico que predomina no Brasil, tem gerado demandas inadequadas. (INCA, 2016; Santos et al., 2019a) 
Diante disso, nota-se uma falha na implementação efetiva das diretrizes nacionais para detecção precoce do câncer que segundo Santos et al., (2019) está relacionada a pouca tradição organizacional no uso das diretrizes, a baixa aceitação por meio dos profissionais e a falta de organização dos serviços de saúde. Dessa forma, o câncer do colo uterino permanece sendo um importante problema de saúde pública no Brasil.

De acordo com dados do Instituto Nacional de Câncer (INCA), essa neoplasia é a quarta mais incidente na população feminina, com predomínio na faixa etária dos 45 aos 50 anos. Além disso, representa a quarta maior causa de mortalidade feminina (INCA, 2019; INCA, 2021a). Estima-se para cada ano do triênio 2020-2022, o total de 16.590 novos casos, com um risco estimado de 15,43 casos a cada 100 mil mulheres. (INCA, 2019)

Frente a magnitude deste problema, é imprescindível compreender a abrangência da cobertura e adesão das diretrizes preconizadas em todo território nacional. Nesse sentido, os indicadores de saúde constituem importantes ferramentas gerenciais, que possibilitam a avaliação dos serviços oferecidos, através da mensuração do alcance da população alvo, da oferta e do acesso ao exame preventivo. Face ao exposto, o presente estudo tem por objetivo analisar os indicadores relacionados à cobertura $\mathrm{e}$ adesão às diretrizes para controle do Câncer de Colo de útero no Brasil no período de 2015 a 2020.

\section{Metodologia}

Trata-se de um estudo ecológico, de abordagem quantitativa, elaborado com base nos dados contidos nos Sistemas de Informação em Saúde: O Sistema de Informação do Câncer (SISCAN) e o Sistema de Informação Ambulatorial (SIA/SUS). Também foram utilizados dados provenientes do censo demográfico do Instituto Brasileiro de Geografia e Estatística (IBGE) e do Instituto Nacional de Câncer (INCA). Para esta pesquisa foram selecionados os seguintes indicadores: Razão de exames citopatológicos do colo do útero em mulheres de 25 a 64 anos e a população feminina da mesma faixa etária, Proporção de exames citopatológicos do colo do útero na faixa etária alvo, Proporção de exames citopatológicos do colo do útero sem citologia anterior e Proporção de exames citopatológicos do colo do útero com periodicidade trienal.

A população de estudo foi composta por todas as mulheres residentes nos estados brasileiros na faixa etária e periodicidade do exame selecionadas no estudo e atendidas nos estabelecimentos de saúde com natureza jurídica pública ou privada (presta serviço ao Sistema Único de Saúde em caráter complementar). A análise dos dados foi realizada por meio do cálculo dos indicadores selecionados no programa Excel, os resultados foram dispostos na forma de tabelas.

A oferta de exames preventivos para a população feminina foi avaliada através do indicador da Razão de exames citopatológicos do colo do útero em mulheres de 25 a 64 anos e a população feminina da mesma faixa etária que é representado pela relação entre o total de exames realizados em mulheres de 25 a 64 anos e um terço das mulheres deste mesmo grupo etário residentes no mesmo local e período. Os respectivos dados foram consultados no IBGE e no SIA/SUS. De acordo com o INCA o parâmetro para análise será um. (INCA, 2014)

A adequação do acesso ao exame preventivo pela população alvo foi avaliada por meio do indicador de Proporção de exames citopatológicos do colo do útero na faixa etária alvo, que foi calculado através da razão entre o número de exames citopatológicos do colo do útero em mulheres na faixa etária de 25 a 64 anos, residentes em determinado local e período multiplicado por 100, e o número de exames citopatológicos do colo do útero em mulheres de todas as idades, residentes no respectivo local e período. De acordo com o INCA, o parâmetro para análise será de 80\%. (INCA, 2014)

A capacidade da rede assistencial em captar mulheres que realizarão o exame citológico pela primeira vez foi avaliada pelo indicador de Proporção de exames citopatológicos do colo do útero sem citologia anterior, que foi calculado através da razão entre número de exames citopatológicos do colo do útero sem relato de exame anterior, em mulheres na faixa etária de 25 a 64 anos, residentes em determinado local e período multiplicado por 100 e o número de exames citopatológicos do colo do útero em mulheres de 25 a 64 anos, residentes no respectivo local e período. Não há parâmetro de análise definido para este 
indicador. (INCA, 2014)

A adequação da realização do exame preventivo pela população feminina na periodicidade recomendada (trienal) foi avaliada pelo indicador de Proporção de exames citopatológicos do colo do útero com periodicidade trienal, que foi calculado através da razão entre o número de exames citopatológicos do colo do útero com relato de exame anterior há três anos, em mulheres na faixa etária de 25 a 64 anos, residentes em determinado local e ano multiplicado por 100 e o número de exames citopatológicos do colo do útero com relato de exame anterior em mulheres de 25 a 64 anos, residentes no respectivo local e ano. De acordo com o INCA, o parâmetro para análise será de $85 \%$. (INCA, 2014)

O presente estudo foi submetido ao comitê de ética em pesquisa, do centro de ciências médicas da Universidade Federal Fluminense, e aprovado sob o CAAE: 47681121.4.0000.8160, parecer de número 4.768.979.

\section{Resultados}

No Brasil foram registrados, no âmbito do Sistema Único de Saúde, 13.246.584 exames citopatológicos no período de 2015 a 2020 na faixa etária de 25 a 64 anos. Na análise do indicador de Razão de exames citopatológicos do colo do útero em mulheres de 25 a 64 anos e a população feminina da mesma faixa etária verificou-se, no período estudado, uma tendência de queda em todas as regiões do país. O maior valor $(0,249)$ foi observado em 2015 na região sudeste e o menor valor $(0,007)$ em 2019 na região norte (Tabela 1).

Tabela 1 - Razão de exames citopatológicos do colo do útero em mulheres de 25 a 64 anos e a população feminina da mesma faixa etária por região no período de 2015 a 2020.

\begin{tabular}{lcccccc}
\hline Região & 2015 & 2016 & 2017 & 2018 & 2019 & 2020 \\
\hline Norte & 0,200 & 0,160 & 0,156 & 0,093 & 0,007 & 0,053 \\
Nordeste & 0,153 & 0,102 & 0,042 & 0,026 & 0,022 & 0,013 \\
Sudeste & 0,249 & 0,207 & 0,170 & 0,173 & 0,152 & 0,094 \\
Sul & 0,100 & 0,087 & 0,066 & 0,045 & 0,024 & 0,011 \\
Centro Oeste & 0,079 & 0,056 & 0,071 & 0,034 & 0,040 & 0,020 \\
\hline
\end{tabular}

Fonte: Ministério da Saúde - Sistema de Informações Ambulatoriais do SUS (SIA/SUS) e Instituto Brasileiro de Geografia e Estatística (IBGE) (Data de acesso: 06/12/2021).

Em relação ao mesmo indicador, na avaliação por estados observou-se que o parâmetro de análise não foi alcançado no período estudado. Os maiores valores foram encontrados no Amazonas (2015: 0,435; 2016: 0,418; 2017: 0,458; 2018: 0,304; 2019: 0,284; 2020: 0,167), padrão semelhante foi observado no Piauí (2015: 0,503; 2016: 0,425; 2017: 0,466; 2018: 0,288; 2019 : 0,258), exceto em 2020, onde o maior valor foi registrado em São Paulo (2020: 0,154). Os menores valores oscilaram entre Roraima (2015: 0,001; 2016: 0,002; 2017: 0,001; 2018: 0,001; 2019: 0,002), Amapá (2015: 0,001; 2016: 0,002; 2017: 0,002; 2018:0,001), Sergipe (2016: 0,002), Paraíba (2019: 0,002; 2020: 0,001) e Rio Grande do Norte (2020: 0,002). (Tabela 2) 
Tabela 2 - Razão de exames citopatológicos do colo do útero em mulheres de 25 a 64 anos e a população feminina da mesma faixa etária por estados no período de 2015 a 2020.

\begin{tabular}{|c|c|c|c|c|c|c|}
\hline Unidade da Federação & 2015 & 2016 & 2017 & 2018 & 2019 & 2020 \\
\hline Rondônia & 0,305 & 0,287 & 0,334 & 0,166 & 0,151 & 0,042 \\
\hline Acre & 0,092 & 0,004 & 0,004 & 0,003 & 0,039 & 0,098 \\
\hline Amazonas & 0,435 & 0,418 & 0,458 & 0,304 & 0,284 & 0,167 \\
\hline Roraima & 0,001 & 0,002 & 0,001 & 0,001 & 0,002 & 0,003 \\
\hline Pará & 0,147 & 0,083 & 0,047 & 0,020 & 0,015 & 0,005 \\
\hline Amapá & 0,001 & 0,002 & 0,002 & 0,001 & 0,057 & 0,130 \\
\hline Tocantins & 0,005 & 0,005 & 0,007 & 0,009 & 0,018 & 0,012 \\
\hline Maranhão & 0,080 & 0,028 & 0,004 & 0,005 & 0,006 & 0,002 \\
\hline Piauí & 0,503 & 0,425 & 0,466 & 0,288 & 0,258 & 0,109 \\
\hline Ceará & 0,399 & 0,259 & 0,011 & 0,007 & 0,007 & 0,005 \\
\hline Rio Grande do Norte & 0,025 & 0,006 & 0,004 & 0,004 & 0,003 & 0,002 \\
\hline Paraíba & 0,036 & 0,005 & 0,004 & 0,002 & 0,002 & 0,001 \\
\hline Pernambuco & 0,015 & 0,040 & 0,008 & 0,012 & 0,010 & 0,004 \\
\hline Alagoas & 0,140 & 0,141 & 0,047 & 0,027 & 0,005 & 0,002 \\
\hline Sergipe & 0,006 & 0,002 & 0,007 & 0,008 & 0,010 & 0,008 \\
\hline Bahia & 0,133 & 0,063 & 0,031 & 0,010 & 0,009 & 0,015 \\
\hline Minas Gerais & 0,319 & 0,144 & 0,025 & 0,013 & 0,013 & 0,009 \\
\hline Espírito Santo & 0,066 & 0,023 & 0,018 & 0,014 & 0,005 & 0,004 \\
\hline Rio de Janeiro & 0,127 & 0,121 & 0,114 & 0,126 & 0,120 & 0,055 \\
\hline São Paulo & 0,279 & 0,285 & 0,271 & 0,277 & 0,241 & 0,154 \\
\hline Paraná & 0,010 & 0,008 & 0,010 & 0,009 & 0,011 & 0,008 \\
\hline Santa Catarina & 0,063 & 0,084 & 0,034 & 0,023 & 0,011 & 0,009 \\
\hline Rio Grande do Sul & 0,209 & 0,165 & 0,138 & 0,093 & 0,044 & 0,015 \\
\hline Mato Grosso do Sul & 0,101 & 0,120 & 0,154 & 0,148 & 0,182 & 0,080 \\
\hline Mato Grosso & 0,020 & 0,009 & 0,014 & 0,013 & 0,014 & 0,005 \\
\hline Goiás & 0,010 & 0,010 & 0,0108 & 0,014 & 0,010 & 0,007 \\
\hline Distrito Federal & 0,269 & 0,148 & 0,190 & 0,003 & 0,009 & 0,010 \\
\hline BRASIL & 0,185 & 0,147 & 0,113 & 0,099 & 0,086 & 0,051 \\
\hline
\end{tabular}

Fonte: Ministério da Saúde - Sistema de Informações Ambulatoriais do SUS (SIA/SUS) e Instituto Brasileiro de Geografia e Estatística (IBGE) (Data de acesso: 06/12/2021).

No que se refere ao indicador de proporção de exames citopatológicos do colo do útero na faixa etária alvo, verificouse que durante o intervalo considerado, as maiores proporções foram alcançadas pelas regiões norte e sudeste, e as menores proporções foram registradas nas regiões Sul e Centro Oeste. Cabe ressaltar que a região Sul apresentou uma queda expressiva ao longo dos anos (Tabela 3 ). 
Tabela 3 - Proporção de exames citopatológicos do colo do útero na faixa etária alvo por região no período de 2015 a 2020.

\begin{tabular}{lcccccc}
\hline Região & 2015 & 2016 & 2017 & 2018 & 2019 & 2020 \\
\hline Norte & 73 & 69 & 67 & 55 & 54 & 59 \\
Nordeste & 62 & 54 & 33 & 22 & 20 & 23 \\
Sudeste & 72 & 68 & 66 & 67 & 66 & 68 \\
Sul & 44 & 41 & 35 & 28 & 18 & 16 \\
Centro Oeste & 48 & 43 & 48 & 33 & 35 & 37 \\
\hline
\end{tabular}

Fonte: Ministério da Saúde - Sistema de Informações Ambulatoriais do SUS (SIA/SUS) e Instituto Brasileiro de Geografia e Estatística (IBGE) (Data de acesso: 06/12/2021).

Na região Norte, os estados que atingiram as maiores proporções foram Amazonas (2015: 78,839; 2016: 79,127; 2017: 78,995; 2018: 73,524; 2019: 77,423; 2020: 80,279), Rondônia (2015:78,805; 2016: 79,085; 2017: 81,797; 2018:70,217; 2019: 64,928) salienta-se, que o Amazonas alcançou a meta em 2020 e Rondônia em 2017. Ainda nessa região, os menores valores foram observados de forma consecutiva no estado do Roraima (2015: 2,177; 2016: 2,706; 2017: 1,331; 2018: 1,035; 2019: 1,827; 2020: 4,969), e em alternância nos estados de Tocantins (2015: 5,264), Acre (2016: 2,895; 2017: 2,995), Amapá (2018: 1,844) e Pará (2019: 17,008; 2020: 13,062). Na região Sudeste, os maiores valores foram identificados no Rio de Janeiro (2015: 73,017; 2016: 72,508; 2017: 73,493; 2018: 73,074; 2019: 72,372; 2020: 70,302), Minas Gerais (2015: 73,981) e São Paulo (2016: 71,537; 2017: 72,193; 2018: 73,425; 2019: 73,498; 2020: 74,654), os menores valores foram registrados nos estados de Espírito Santo (2015: 36,650; 2016: 15,957; 2017: 14,238; 2018: 11,160; 2019: 4,144; 2020: 7,209) e Minas Gerais (2016: 59,665; 2017: 21,317; 2018: 12,557; 2019: 14,130; 2020: 17,944). Verificou-se uma tendência de queda significativa nos seguintes estados: Piauí, Ceará, Alagoas e Bahia. Destaca-se a região Sul com tendência de queda em todo o período estudado (Tabela 4).

Tabela 4 - Proporção de exames citopatológicos do colo do útero na faixa etária alvo por estados no período de 2015 a 2020.

\begin{tabular}{lcccccc}
\hline Unidade da Federação & 2015 & 2016 & 2017 & 2018 & 2019 & 2020 \\
\hline Rondônia & 78,805 & 79,085 & 81,797 & 70,217 & 64,928 & 56,126 \\
Acre & 43,123 & 2,895 & 2,995 & 2,309 & 24,898 & 67,699 \\
Amazonas & 78,839 & 79,127 & 78,995 & 73,524 & 77,423 & 80,279 \\
Roraima & 2,177 & 2,706 & 1,331 & 1,035 & 1,827 & 4,969 \\
Pará & 73,629 & 61,140 & 45,280 & 23,726 & 17,008 & 13,062 \\
Amapá & 66,176 & 4,475 & 5,528 & 1,844 & 57,994 & 92,260 \\
Tocantins & 5,264 & 7,420 & 8,954 & 10,354 & 29,831 & 20,480 \\
Maranhão & 58,496 & 33,325 & 5,778 & 6,678 & 7,497 & 6,310 \\
Piauí & 77,438 & 77,886 & 80,545 & 73,626 & 71,006 & 72,468 \\
Ceará & 77,363 & 74,338 & 13,183 & 8,314 & 8,120 & 10,113 \\
Rio Grande do Norte & 21,739 & 6,561 & 3,623 & 3,973 & 3,893 & 3,799 \\
Paraíba & 26,490 & 4,991 & 3,435 & 2,075 & 1,985 & 2,645 \\
Pernambuco & 13,018 & 27,986 & 7,474 & 10,270 & 9,964 & 8,801 \\
Alagoas & 62,631 & 60,369 & 32,666 & 19,171 & 4,296 & 4,023 \\
Sergipe & 4,930 & 2,272 & 7,145 & 7,142 & 9,853 & 15,715
\end{tabular}




\begin{tabular}{lcccccc} 
Bahia & 61,959 & 43,487 & 27,698 & 10,732 & 10,114 & 27,110 \\
Minas Gerais & 73,981 & 59,665 & 21,317 & 12,557 & 14,130 & 17,944 \\
Espírito Santo & 36,650 & 15,957 & 14,238 & 11,160 & 4,144 & 7,209 \\
Rio de Janeiro & 73,017 & 72,508 & 73,493 & 73,074 & 72,372 & 70,302 \\
São Paulo & 71,645 & 71,537 & 72,193 & 73,425 & 73,498 & 74,654 \\
Paraná & 6,974 & 5,571 & 7,126 & 6,741 & 8,266 & 11,692 \\
Santa Catarina & 32,502 & 37,940 & 21,113 & 15,662 & 9,666 & 14,065 \\
Rio Grande do Sul & 64,936 & 59,912 & 56,062 & 48,685 & 30,669 & 20,037 \\
Mato Grosso do Sul & 42,257 & 50,606 & 60,412 & 59,882 & 65,207 & 65,480 \\
Mato Grosso & 16,686 & 7,808 & 11,434 & 11,381 & 13,161 & 8,841 \\
Goiás & 13,860 & 14,410 & 13,918 & 17,923 & 13,552 & 19,456 \\
Distrito Federal & 78,740 & 78,680 & 79,529 & 13,506 & 15,281 & 35,717 \\
\hline
\end{tabular}

Fonte: Ministério da Saúde - Sistema de Informação do Câncer (SISCAN) e Instituto Brasileiro de Geografia e Estatística (IBGE) (Data de acesso: 06/12/2021).

$\mathrm{Na}$ análise da proporção de exames citopatológicos do colo do útero sem citologia anterior, observou-se um padrão de queda em todas as regiões do Brasil. As maiores proporções foram identificadas nas regiões norte (2016: 18; 2018: 18; 2019: 15; 2020: 13) e no Nordeste (2015:18; 2017: 20) (Tabela 5).

Tabela 5 - Proporção de exames citopatológicos do colo do útero sem citologia anterior por região no período de 2015 a 2020.

\begin{tabular}{lcccccc}
\hline Região & 2015 & 2016 & 2017 & 2018 & 2019 & 2020 \\
\hline Norte & 18 & 18 & 16 & 16 & 15 & 13 \\
Nordeste & 18 & 17 & 20 & 16 & 14 & 11 \\
Sudeste & 16 & 14 & 13 & 12 & 11 & 10 \\
Sul & 12 & 11 & 10 & 9 & 9 & 8 \\
Centro Oeste & 14 & 14 & 14 & 13 & 13 & 11 \\
\hline
\end{tabular}

Fonte: Ministério da Saúde - Sistema de Informação do Câncer (SISCAN) e Instituto Brasileiro de Geografia e Estatística (IBGE) (Data de acesso: 06/12/2021).

Nessas regiões destacam-se com maiores valores os estados do Pará, Amapá, Maranhão e do Alagoas. Os menores valores foram registrados na região Sul, que variou de 12 em 2015 a 8 em 2020, o estado de Santa Catarina se sobressai com os menores valores nos cinco anos consecutivos (Tabela 6). 
Tabela 6 - Proporção de exames citopatológicos do colo do útero sem citologia anterior por estados no período de 2015 a 2020.

\begin{tabular}{|c|c|c|c|c|c|c|}
\hline UF de residência & 2015 & 2016 & 2017 & 2018 & 2019 & 2020 \\
\hline Rondônia & 16 & 18 & 16 & 14 & 15 & 14 \\
\hline Acre & 13 & 14 & 12 & 12 & 12 & 10 \\
\hline Amazonas & 16 & 16 & 15 & 15 & 15 & 13 \\
\hline Roraima & 12 & 33 & 11 & 10 & 12 & 12 \\
\hline Pará & 21 & 21 & 18 & 18 & 16 & 14 \\
\hline Amapá & 22 & 18 & 25 & 37 & 26 & 23 \\
\hline Tocantins & 16 & 11 & 11 & 11 & 10 & 11 \\
\hline Maranhão & 32 & 27 & 25 & 25 & 21 & 16 \\
\hline Piauí & 19 & 26 & 24 & 25 & 21 & 17 \\
\hline Ceará & 31 & 24 & 17 & 17 & 16 & 13 \\
\hline Rio Grande do Norte & 14 & 13 & 11 & 11 & 11 & 10 \\
\hline Paraíba & 18 & 17 & 13 & 11 & 10 & 8 \\
\hline Pernambuco & 14 & 12 & 11 & 10 & 9 & 8 \\
\hline Alagoas & 24 & 26 & 32 & 26 & 19 & 14 \\
\hline Sergipe & 15 & 15 & 14 & 14 & 13 & 9 \\
\hline Bahia & 15 & 15 & 14 & 15 & 12 & 11 \\
\hline Minas Gerais & 18 & 14 & 13 & 12 & 10 & 9 \\
\hline Espírito Santo & 11 & 11 & 10 & 8 & 7 & 6 \\
\hline Rio de Janeiro & 18 & 17 & 14 & 15 & 17 & 22 \\
\hline São Paulo & 15 & 14 & 13 & 12 & 11 & 10 \\
\hline Paraná & 14 & 12 & 10 & 8 & 8 & 7 \\
\hline Santa Catarina & 9 & 9 & 9 & 8 & 8 & 7 \\
\hline Rio Grande do Sul & 11 & 10 & 11 & 9 & 10 & 9 \\
\hline Mato Grosso do Sul & 8 & 8 & 8 & 8 & 8 & 7 \\
\hline Mato Grosso & 14 & 13 & 13 & 11 & 10 & 8 \\
\hline Goiás & 19 & 18 & 18 & 17 & 16 & 14 \\
\hline Distrito Federal & 21 & 24 & 24 & 22 & 19 & 17 \\
\hline
\end{tabular}

Fonte: Ministério da Saúde - Sistema de Informação do Câncer (SISCAN) (Data de acesso: 06/12/2021).

Em relação a proporção de exames citopatológicos do colo do útero com periodicidade trienal, as regiões Norte e Nordeste apresentaram os melhores desempenhos, não alcançaram a meta pactuada apenas ano de 2018. As regiões Sudeste, Sul e Centro-Oeste registraram valores inferiores ao parâmetro de análise no período de 2018 a 2020 (Tabela 7). 
Tabela 7 - Proporção de exames citopatológicos do colo do útero com periodicidade trienal por região no período de 2015 a 2020 .

\begin{tabular}{lcccccc}
\hline Região & 2015 & 2016 & 2017 & 2018 & 2019 & 2020 \\
\hline Norte & 88 & 87 & 86 & 84 & 85 & 86 \\
Nordeste & 89 & 89 & 85 & 83 & 85 & 85 \\
Sudeste & 86 & 87 & 85 & 82 & 82 & 82 \\
Sul & 87 & 88 & 85 & 83 & 82 & 82 \\
Centro Oeste & 87 & 88 & 86 & 82 & 81 & 83 \\
\hline
\end{tabular}

Fonte: Ministério da Saúde - Sistema de Informação do Câncer (SISCAN) e Instituto Brasileiro de Geografia e Estatística (IBGE) (Data de acesso: 06/12/2021)

Na análise por estado, Amapá foi o único a não alcançar a meta em 2015 (80), seguiu abaixo do padrão em 2016 (81) e 2017(83) apesar de obter resultados melhores. Distrito Federal manteve resultado inferior à meta de 2016 a 2020 . Num contexto geral os anos de 2018 (17), 2019 (17) e 2020 (15) concentraram um maior quantitativo de estados abaixo do padrão preconizado (Tabela 8).

Tabela 8 - Proporção de exames citopatológicos do colo do útero com periodicidade trienal por estados no período de 2015 a 2020.

\begin{tabular}{|c|c|c|c|c|c|c|}
\hline UF de residência & 2015 & 2016 & 2017 & 2018 & 2019 & 2020 \\
\hline Rondônia & 86 & 86 & 82 & 85 & 86 & 87 \\
\hline Acre & 90 & 89 & 90 & 88 & 84 & 84 \\
\hline Amazonas & 90 & 87 & 91 & 87 & 88 & 89 \\
\hline Roraima & 85 & 89 & 91 & 85 & 82 & 84 \\
\hline Pará & 87 & 86 & 86 & 84 & 85 & 86 \\
\hline Amapá & 80 & 81 & 83 & 87 & 85 & 89 \\
\hline Tocantins & 90 & 89 & 86 & 82 & 85 & 84 \\
\hline Maranhão & 89 & 90 & 81 & 80 & 82 & 87 \\
\hline Piauí & 89 & 89 & 88 & 87 & 88 & 90 \\
\hline Ceará & 86 & 87 & 84 & 83 & 87 & 85 \\
\hline Rio Grande do Norte & 88 & 89 & 85 & 81 & 83 & 84 \\
\hline Paraíba & 91 & 92 & 91 & 87 & 87 & 88 \\
\hline Pernambuco & 90 & 92 & 97 & 85 & 85 & 86 \\
\hline Alagoas & 87 & 89 & 87 & 85 & 86 & 85 \\
\hline Sergipe & 87 & 89 & 86 & 82 & 84 & 85 \\
\hline Bahia & 87 & 86 & 85 & 83 & 83 & 82 \\
\hline Minas Gerais & 87 & 88 & 85 & 82 & 83 & 81 \\
\hline Espírito Santo & 88 & 89 & 87 & 84 & 82 & 85 \\
\hline Rio de Janeiro & 87 & 88 & 84 & 81 & 80 & 83 \\
\hline São Paulo & 87 & 88 & 85 & 82 & 81 & 82 \\
\hline
\end{tabular}




\begin{tabular}{|c|c|c|c|c|c|c|}
\hline Paraná & 86 & 88 & 85 & 83 & 82 & 83 \\
\hline Santa Catarina & 89 & 89 & 86 & 82 & 81 & 82 \\
\hline Rio Grande do Sul & 97 & 88 & 87 & 85 & 84 & 84 \\
\hline Mato Grosso do Sul & 90 & 92 & 88 & 84 & 84 & 84 \\
\hline Mato Grosso & 89 & 89 & 86 & 83 & 83 & 84 \\
\hline Goiás & 85 & 87 & 85 & 81 & 81 & 84 \\
\hline Distrito Federal & 86 & 83 & 84 & 78 & 77 & 83 \\
\hline
\end{tabular}

Fonte: Ministério da Saúde - Sistema de Informação do Câncer (SISCAN) (Data de acesso: 06/12/2021).

\section{Discussão}

O indicador de Razão de exames citopatológicos do colo do útero em mulheres de 25 a 64 anos e a população feminina da mesma faixa etária representa uma aproximação da cobertura de exames. O conhecimento dessa razão contribui para a correlação entre oferta e demanda, possibilita a identificação de disparidades, além de fornecer subsidio para processos de planejamento, gestão e avaliação de políticas públicas na área de saúde da mulher. (Santos et al., 2021)

A análise deste indicador revelou uma baixa oferta do exame citopatológico para a população alvo, que reflete numa cobertura inferior a recomendada pelo Ministério da Saúde. Para Lopes \& Ribeiro (2019) barreiras organizacionais e disparidades socioeconômicas, culturais e raciais, representam fatores de interferência no rastreamento. Segundo Morais et al., (2017) baixas coberturas estão relacionadas a dificuldades de acesso ao exame citopatológico no SUS, como por exemplo obstáculos no agendamento de consulta com um profissional da saúde. Todavia, deve-se salientar que a cobertura deste indicador se restringe a população feminina que realiza o exame citopatológico no SUS, portanto, baixas razões podem evidenciar uma maior cobertura da saúde suplementar. (Santos et al., 2012)

Ressalta-se que nenhuma das regiões alcançou o parâmetro nacional estipulado pelo Ministério da Saúde, todas obtiveram resultado inferior a um. Corroborando com o exposto, um estudo que avaliou a produção de procedimentos da linha de cuidado do câncer do colo do útero no Sistema Único de Saúde do Brasil em 2015, constatou que a produção de exames de rastreamento foi inferior a necessária para atender às demandas da população-alvo em todas as grandes regiões do país, os maiores déficits de procedimentos foram nas regiões Norte, Centro-Oeste e Nordeste. (Ribeiro et al., 2018)

Além disso, observou-se grandes diferenças regionais e inter-regionais no rastreamento do colo do útero. Neste estudo a região sudeste foi a que mais se aproximou da meta preconizada, enquanto as regiões centro-oeste e nordeste foram as que mais se distanciaram. Um estudo, realizado com dados levantados pelo Vigitel em 2019, identificou que a persistência de desigualdades regionais na realização do exame citopatológico, afetam sobretudo mulheres residentes na região Nordeste do país. (Schäfer et al., 2021) Conforme Bezerra et al., (2021) para deter iniquidades inter e intrarregionais é fundamental aprimorar o acesso e a oferta dos serviços realizados na atenção básica.

Para além da oferta e realização do Papanicolau, Santos et al., (2019b) consideram que para aumentar a cobertura do exame citopatológico, faz-se necessário que o enfermeiro na Estratégia da Saúde da Família (ESF) realize busca ativa das mulheres, promoção de vínculos, educação em saúde e se articule com representantes da comunidade. Rocha et al., (2020) defendem que a consulta de enfermagem realizada de forma adequada é imprescindível para a prevenção e detecção precoce do câncer, além de representar uma importante aliada ao programa de rastreamento devido ao seu potencial educativo.

No que tange ao indicador de proporção de exames citopatológicos do colo do útero na faixa etária alvo, sua análise expressa a adequação do acesso ao exame preventivo pela população-alvo. O Ministério da Saúde, estipula um parâmetro de $80 \%$ para análise do mesmo. Contudo, todas as regiões permaneceram abaixo deste parâmetro. Tendo em vista um programa de 
rastreamento organizado com cobertura de $100 \%$ da população-alvo, estimou-se que a necessidade de exames citopatológicos de rastreamento corresponde a 39,8\% das mulheres na faixa etária alvo. (Ribeiro et al., 2019) No entanto, de acordo com Ribeiro et al., (2019) o Brasil ainda não possui um programa de rastreamento organizado, as ações são predominantemente oportunísticas. Isso implica em um grande quantitativo de exames realizados fora da faixa etária preconizada. (Souza et al., 2018; Corrêa et al., 2017; Ribeiro et al., 2020)

Além disso, há de se considerar os motivos que influenciam as mulheres a não realizarem o exame. Nesse tocante notase que a falta de conhecimento sobre a importância da realização do exame preventivo para o rastreamento do câncer do colo está diretamente ligada a não adesão ao exame. E a falta de tempo, a vergonha, o desinteresse, a dificuldade de acesso ao serviço de saúde, o medo do procedimento e de descobrir o câncer são fatores que dificultam a realização do mesmo. (Silva, et al., 2021)

As regiões Norte e Nordeste apresentaram o melhor desempenho na captação de mulheres sem citologia anterior, indicativo de que mulheres que nunca haviam realizado o exame estão sendo alcançadas. Resultado similar foi observado no estudo de Santos et al., (2012) que analisou a distribuição espacial dos indicadores pactuados para o câncer do colo do útero no Brasil, no período de 2006 a 2009, e identificou um elevado percentual de captação de mulheres sem citologia anterior nas regiões Norte, Nordeste e Centro Oeste.

A região Sul apresentou os menores valores do indicador supracitado. Para Santos et al., (2012) proporções baixas podem denotar uma menor capacidade da rede assistencial na captação e rastreamento, e em alguns casos indicam a inadequação da oferta de exames, baixa capacidade de sensibilização e dificuldades de acesso geográfico aos serviços ou, por outro lado, podem evidenciar uma boa cobertura do programa, que tem como resultado a baixa captação de mulheres sem citologia anterior. Por este motivo, faz-se necessário relacionar esse indicador com a cobertura de cada estado, em estados cuja cobertura é baixa, espera-se seu aumento progressivo, decorrente da captação de mulheres do grupo etário alvo que realizarão o exame pela primeira vez. (INCA, 2014)

O indicador de proporção de exames citopatológicos do colo do útero com periodicidade trienal, refere-se quantidade de exames realizados a cada três anos entre o total de exames realizados no grupo alvo. Em discordância da recomendação de realização do exame citopatológico em intervalo trienal, estudos apontam um elevado número de exames realizados em intervalos de um ano ou menos. (Ribeiro et al., 2020; Pereira Filho et al., 2021) Para Lopes \& Ribeiro (2019), a falta de conhecimento sobre a periodicidade adequada constitui uma barreira ao seu cumprimento, relatam também que as disparidades socioeconômicas e demográficas condicionam a cobertura e a periodicidade adequada dos exames preventivos.

No presente estudo observou-se que todas as regiões alcançaram o parâmetro de análise (85\%) no período de 2015 a 2017, entretanto em 2018 todas obtiveram resultado inferior, nos anos de 2019 e 2020 somente as regiões norte e nordeste voltaram a atingir o parâmetro estipulado. Todavia deve-se considerar o viés de informação, visto que tal indicador sofre interferência da prevalência de lesões e pela captação das mulheres para o rastreamento. (INCA, 2014)

Este estudo apresenta limitações devido a utilização de dados secundários, provenientes dos Sistemas de Informação em Saúde. Cabe ressaltar que no SISCAN "a confiabilidade das informações geradas depende da qualidade dos dados coletados e registrados." (INCA, 2021b) "Tendo em vista que o SIA mantém diversos instrumentos de registro, o nível de detalhamento das informações disponíveis neste sistema irá depender diretamente do instrumento no qual os procedimentos são registrados." (Ministério da Saúde, 2015)

\section{Conclusão}

Diante dos achados, conclui-se que as tendências de queda evidenciadas nas análises dos indicadores de cobertura e adesão às diretrizes nacionais podem refletir no aumento da incidência, morbidade e mortalidade do câncer do colo do útero, contrariando o atributo essencial dos programas de rastreamento que visam o diagnóstico precoce. 
Por meio deste trabalho foi possível contribuir com reflexões acerca da importância da atuação do Enfermeiro na linha de cuidado do câncer do colo do útero, sobretudo no que concerne ao rastreamento e diagnóstico precoce, haja visto que a consulta de enfermagem constitui o cenário ideal para a educação em saúde e a sensibilização das mulheres sobre a importância da adesão ao exame citopatológico na faixa etária e periodicidade adequadas. Ademais, os dados elencados nesse estudo podem orientar gestores de saúde no planejamento de ações de monitoramento e detecção precoce do câncer do colo uterino, bem como na mensuração da eficiência das estratégias já adotadas.

Espera-se que o panorama epidemiológico apresentado neste estudo, se torne subsídio para o planejamento de novas estratégias voltadas para o monitoramento e a prevenção desta patologia, bem como o redirecionamento e fortalecimento das políticas públicas já existentes.

\section{Referências}

Bezerra, H. S., Mendes, T. M. C., Souza, T. A., Nascimento, A. K. F., Macedo, H. K. S., \& Andrade, F. B. (2021). Cobertura da triagem para câncer do colo do útero em um estado do nordeste do Brasil. Journal of Human Growth and Development, 31(1), 145-151. https://dx.doi.org/10.36311/jhgd.v31.10319

Corrêa, C. S. L., Lima, A. S., Leite, I. C. G., Pereira, L. C., Nogueira, M. C., Duarte, D. A. P., Fayer, V. A., \& Bustamante-Teixeira, M. T. (2017). Rastreamento do câncer do colo do útero em minas gerais: avaliação a partir de dados do sistema de informação do câncer do colo do útero (SISCOLO). Cadernos Saúde Coletiva, 25 (3), 315-323. https://doi.org/10.1590/1414-462X201700030201

Instituto Nacional de Câncer José Alencar Gomes da Silva. (2014). Ficha técnica de indicadores das ações de controle do câncer do colo do útero. https://www.inca.gov.br/sites/ufu.sti.inca.local/files//media/document//fichatecnicaindicadorescolo14.pdf

Instituto Nacional de Câncer José Alencar Gomes da Silva. (2016). Diretrizes Brasileiras para o Rastreamento do Câncer do Colo do Útero, 2. ed. rev. atual., http://www.citologiaclinica.org.br/site/pdf/documentos/diretrizes-para-o-rastreamento-do-cancer-do-colo-do-utero_2016.pdf

Instituto Nacional de Câncer José Alencar Gomes da Silva. (2019). Estimativa 2020: incidência de câncer no Brasil. https://www.inca.gov.br/publicacoes/livros/estimativa-2020-incidencia-decancer-no-brasil

Instituto Nacional de Câncer José Alencar Gomes da Silva. (2021a). Conceito e Magnitude. https://www.inca.gov.br/controle-do-cancer-do-colo-doutero/conceito-e-magnitude

Instituto Nacional de Câncer José Alencar Gomes da Silva. (2021b). Qualidade dos dados do Sistema de Informação do Câncer (Siscan) - 2016 a 2020. https://www.inca.gov.br/sites/ufu.sti.inca.local/files//media/document//relatorio_qualidade_dados_siscan_maio_2021.pdf

Lopes, V. A. S., \& Ribeiro, J. M. (2019). Fatores limitadores e facilitadores para o controle do câncer de colo de útero: uma revisão de literatura. Ciência \& Saúde Coletiva, 24(9), 3431-3442. https://doi.org/10.1590/1413-81232018249.32592017

Ministério da Saúde. (2015). Sistemas de Informação da Atenção à Saúde: Contextos Históricos, Avanços e Perspectivas no SUS. https://bvsms.saude.gov.br/bvs/publicacoes/sistemas_informacao_atencao_saude_contextos_historicos.pdf

Morais, A. L. J., Passos, T. S., Santos, D. M. S., Vargas, M. M., \& Oliveira, C. C. C. (2017). Percepção de mulheres sobre a atenção primária no âmbito da política do câncer de colo uterino no estado de Sergipe. Ciência, Cuidado $e$ Saúde, 16(2). https://periodicos.uem.br/ojs/index.php/CiencCuidSaude/article/view/22920/pdf

Organização Pan-Americana da Saúde. (2019). HPV e câncer do colo do útero. https://www.paho.org/pt/topicos/hpv-e-cancer-do-colo-do-utero

Pereira Filho, J. L, Azevedo, G. C. A., Theodoro, T.F., Bonfim, BF, Monteiro, P. M., Arouche, R., Anunciação, R. K. L, Pereira, C. L, Buna, S. S. S., Silva, Á. L. S., Araújo, Á. W. M. S., Lima, L. A., Arruda, A. C. P., Santos, T., Silva, A. Z., Abreu, I. C., \& Silva, S. N. (2021). Câncer de colo uterino: análise epidemiológica e citopatológica no Município de São Luís, Estado do Maranhão, Brasil. Research, Society and Development, 10 (8), e33010817074. https://doi.org/10.33448/rsd-v10i8.17074

Ribeiro, B. C., Skonieczny, N. E., Bortoli, C. F.C., \& Massafera, G. I. (2020). Rastreamento do câncer de colo do útero em um município do sudoeste do Paraná. Revista de Saúde Pública do Paraná. 3(1), 41-50. http://revista.escoladesaude.pr.gov.br/index.php/rspp/article/view/370/114

Ribeiro, C. M., \& Silva, G. A. (2018). Avaliação da produção de procedimentos da linha de cuidado do câncer do colo do útero no Sistema Único de Saúde do Brasil em 2015. Epidemiologia e Serviços de Saúde, 27(1), e20172124. https://doi.org/10.5123/S1679-49742018000100004

Ribeiro, C. M., Dias, M. B. K., Pla, M. A. S., Correa, F. M., Russomano, F. B., \& Tomazelli, J. G. (2019). Parâmetros para a programação de procedimentos da linha de cuidado do câncer do colo do útero no Brasil. Cadernos de Saúde Pública, 35(6), e00183118. https://doi.org/10.1590/0102-311X00183118

Rocha, M. D. H. A., Morais, J. B., Andrade, B. B., Cavalcante, P. A. M., Rocha, P. F. A., \& Saiter, R. (2020). Prevenção do câncer de colo de útero na consulta de enfermagem para além do Papanicolau. Revista Cereus, 12(1), 50-63. https://doi.org/10.18605/2175-7275/cereus.v12n1p50-63

Santos, B. L., Costa, S. B. O., Moreira, G. D., Andrade, B. A., Santos, N. A., \& Ximenes, T. P. M. (2021). Análise de citopatologias cérvico-vaginal realizadas na Bahia entre 2015-2019: Indicadores Técnicos. Research, Society and Development, 10(12), e404101220609. https://doi.org/10.33448/rsd-v10i12.20609c 
Research, Society and Development, v. 11, n. 3, e48511326714, 2022

(CC BY 4.0) | ISSN 2525-3409 | DOI: http://dx.doi.org/10.33448/rsd-v11i3.26714

Santos, R. O. M., Ramos, D. N., \& Migowski, A. (2019a). Barreiras na implementação das diretrizes de detecção precoce dos cânceres de mama e colo do útero no Brasil. Physis: Revista de Saúde Coletiva, 29(4). https://doi.org/10.1590/S0103-73312019290402

Santos, R. S., Melo, E. C. P., \& Santos, K. M. (2012). Análise espacial dos indicadores pactuados para o rastreamento do câncer do colo do útero no Brasil. Texto \& Contexto-Enfermagem, 21(4), 800-810. https://www.scielo.br/j/tce/a/RhYf7dHpHR79sddtdTdLgZp/abstract/?lang=pt

Santos, T. L. S., Silveira, M. B., \& Rezende, H. H. A. (2019b). A importância do exame citopatológico na prevenção do câncer do colo uterino. Enciclopédia Biosfera, 16(29), 1947-1961. https://www.conhecer.org.br/enciclop/2019a/sau/a\%20importancia.pdf

Schäfer, A. A., Santos, L. P., Miranda, V. I. A., Tomasi, C. D., Soratto, J., Quadra, M. R., \& Meller, F. O. (2021). Desigualdades regionais e sociais na realização de mamografia e exame citopatológico nas capitais brasileiras em 2019: estudo transversal. Epidemiologia e Serviços de Saúde, 30(4), e2021172. https://doi.org/10.1590/S1679-49742021000400016

Silva, T. R. S., Santos, J. C. M., Oliveira, J. S., Abreu, V. P. L., Silva, R. R., Dantas, K. L. S., Silva, R. M. O., Januário, P. O. S., Gonçalves, G. F. S., Rodrigues, C. F. C., Silva, M. V. F. B., Oliveira, R. T. S., \& Ferreira, R. K. A. (2021). A importância do rastreamento do câncer do colo do útero e fatores relacionados à não adesão. Research, Society and Development, 10 (4), e51710414079. https://doi.org/10.33448/rsd-v10i4.14079

Souza, A. A. R., Silva, M. A. S., Vieira, F. S., Dias H. S. B., Batista, M. R. S., Fernandes, O. T., Cardoso, R. T. L., Araújo, I. C., Matos, M. L. S. S., \& Carvalho, S. (2018). Indicadores de monitoramento do câncer de colo de útero em um Município Maranhense. Revista Eletrônica Acervo Saúde, 11(2), e126. https://doi.org/10.25248/reas.e126.2019

Teixeira, A. S., Rodrigues, F. K. S., Neto, T. C. S., \& Brito, M. A. M. (2021). O exame Papanicolaou como ferramenta para a prevenção do câncer do colo do útero: Revisão Sistemática. Brazilian Journal of Development, 7(11), 105049-105069. https://brazilianjournals.com/index.php/BRJD/article/view/39558/pdf 